\title{
Traumatic brain injury- Possible beneficial effect of omentum
}

\author{
Harry S. Goldsmith* \\ Clinical Professor of Neurological Surgery, University of California, USA
}

\begin{abstract}
Traumatic brain injuries remain one of the most problematic situations presently in neurosurgery. A major problem in this condition is the continued expansion of brain edema which can evidentially cause compression of cerebral arteries. It has been shown that the edema in the spinal cord following injury can be absorbed by the omentum. This paper suggests that the edema in brain injury can also be absorbed by placement of the omentum directly on the injured brain.
\end{abstract}

\section{Background}

Brain edema following trauma remains a serious problem in neurosurgery. The brain edema that develops following injury can lead to compression of cerebral arteries progressing to the death of brain tissue and brain herniation. In 1991, a paper was published that claimed that the omentum does not absorb brain edema and that the omentum has no place in the treatment of traumatic brain injury. This paper being presented will show that the experimental technique employed in demonstrating the lack of omental absorption was flawed.

In studies of the cerebral effects of traumatic brain injury (TBI), the cerebral edema that follows the injury has been shown to be one of the main causes of the morbidity and mortality associated with the injury. The swelling of the brain that occurs in a TBI leads to increased intra-cranial pressure (ICP) that results in compression of cerebral blood vessels which decreases cerebral blood flow, lowers cerebral oxygenation and can lead to brain herniation.

Treatment of cerebral edema following a TBI has been mainly focused on attempts to control the symptoms of the problem rather than on the mechanisms that routinely occur following a TBI. This paper proposes a form of treatment that may have the potential to control the brain edema that is a hallmark of a TBI.

\section{Edema production}

There are two types of brain edema that follow a TBI - cytotoxic and vasogenic. Cytotoxic edema involves water that is intracellular, whereas vasogenic edema is water that is added to brain tissue caused by the disruption of tight endothelial junctions in the capillaries that make up the blood brain barrier (BBB). Disruption of these BBB capillaries allows water to enter extracellular spaces in the brain shortly after injury. The intracellular water content in a cytotoxic neuron is not the early problem following a TBI, but it is vasogenic edema in the brain tissue that is the main problem following a TBI. It has been claimed that the breakage of capillaries in the BBB occurs as early as four to six hours after a TBI and progresses over the next seven days being maximal two to three days after a TBI [1]. Unfortunately, various efforts to treat the results of the ruptured BBB vessels in the first few days following a TBI have not proven successful.

\section{Omental absorption}

This paper suggests that treatment of a TBI could be successful if a vascularized intact omental pedicle is placed directly on the brain following a severe TBI. Any improvement that follows may result from the enormous ability of the omentum to absorb the vasogenic edema that occurs following a TBI.

Early studies of the omentum's absorbability of fluids in humans were first seen in women who developed lymphedema in an extremity following a radical mastectomy for breast cancer. Placing an intact omental pedicle into an involved swollen arm resulted in a reduction of the enlarged edema-filled limb [2]. To further confirm the ability of omental lymphatics to absorb edema fluid, an intact omental pedicle from an animal was placed in a beaker that contained saline. India ink was added to the saline and the blue coloration of the dye appeared in the omental lymphatics within thirty seconds. A publication written by others erroneously reported that the absorption of the India ink occurred in the omental lymphatics within thirty minutes [3]. This statement was incorrect, it was not thirty minutes, it was thirty seconds (Figure 1).

It is suggested that the enormous absorbability of the omentum can be pivotal in the treatment of an acute spinal cord injury (SCI). It is known that the scar that develops after a spinal cord injury is responsible for preventing axons to penetrate through the scar barrier. It has been shown however that when the omentum is placed on a recently injured spinal cord, the scar that routinely occurs is prevented from developing. This is believed to be due to the apparent absorption of fibrinogen which is introduced into the injured spinal cord in massive amounts $[4,5]$. Fibrinogen is the agent that initiates the cascade of physiological activity within the injured spinal cord shortly

Correspondence to: Harry S. Goldsmith, Clinical Professor of Neurological Surgery, University of California, Davis P.O. Box 493, Glenbrook, NV 89413, USA, Tel: 775-749-5801, Fax: 775-749-5861, E-mail: hlgldsmith@aol.com

Key words: Omentum, traumatic brain injury, edema absorption, omental lymphatics

Received: July 06, 2017; Accepted: August 28, 2017; Published: August 31, 2017 


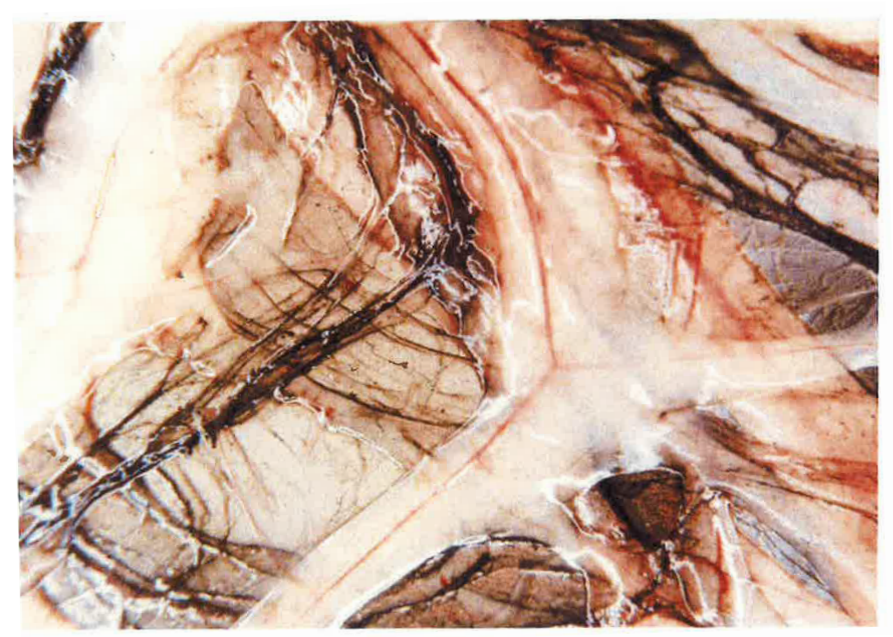

Figure 1. Omental lymphatics showing india ink within 30 seconds of immersion in saline

after injury. When the level of fibrinogen was decreased by omental absorption following an SCI, scar failed to develop in experimental animals [6]. It is known that vasogenic edema can be absorbed by the omentum in an SCI, it seems logical therefore that the omentum can also absorb the vasogenic edema in a TBI.

The physiological mechanisms that allow the omentum to absorb vasogenic edema in the brain following a TBI are based on the lymphatic drainage system that routinely occurs throughout the body. The lymphatic system in the omentum consists of lymphatic vessels and small lymphatic capillaries that connect to the larger lymphatic structures. Key to the absorptive process are thin-wall lymphatic capillaries that have internal valves that control the direction of fluid that passes through the capillaries in only one direction.

When a TBI occurs, there is a rapid infusion of fluid from injured brain tissue that enters the closed-head compartment. The lymphatic capillaries in the brain absorb this fluid from interstitial areas of the brain based on a single physical property: fluid flows from areas of high pressure toward areas of lower pressure. Fluid in the interstitial areas of the brain has a higher pressure after a TBI than the lower pressure found in lymphatic capillaries. This pressure gradient forces fluid through the lymphatic capillaries with the flow propelled in only one direction due to the valves in the lymphatic capillaries. The fluid (lymph), which drains through the lymphatic capillaries into the larger lymphatic vessels, eventually enters the venous system leading to lymph distribution throughout the general circulation. These reactions explain how the omentum can absorb increasing amounts of vasogenic edema in the brain following a TBI.

\section{The omentum for TBI}

Over the past quarter of a century, there has been increasing interest in using the omentum for a host of neurological conditions, such as stroke [7,8], Alzheimer disease [9,10] cerebral palsy [11], etc. In spite of the use of the omentum in the treatment of a variety of clinical conditions, there has been little, if any, interest in the omentum for the absorption of vasogenic edema in the treatment of a TBI. To evaluate the absorbability of this material by the omentum in the treatment of a TBI, the underlying paragraph could be the reason for the failure.

In 1991, a paper was published entitled, "Acute Effects of a Pedicle Omental Graft on Cold Induced Edema in Cats [10]." Vasogenic edema was believed to have been produced by hypothermia in nine cats using a $0.9 \mathrm{~cm}$ cryosurgical probe which was simply placed on the intact dura of cats at a temperature of $-30^{\circ} \mathrm{C}$ for three minutes. Prior to the hypothermic application, a left frontoparietal craniotomy of $2.0 \mathrm{~cm}$ diameter was performed. In the five experimental cats, an intact vascularized omental flap was created from its attachment to the greater curvature of the stomach and brought up to the brain [12] were it was placed in the five experimental cats.

Nothing was done in the four control cats other than applying hypothermic cooling on the surface of the dura, followed by the opening of the dura and arachnoid. The five experimental cats underwent the identical cryosurgical application that had been performed in control cats except that the tip of the intact omental pedicle that had been brought up to the brain was inserted through the craniotomy opening in the skull. This allowed a small piece of the intact omental pedicle to be placed in direct contact with the underlying brain.

The area in the brain that sustained the cryo-application [3] was clearly demarcated as a reddish area which measured $12 \mathrm{~mm}$ to $15 \mathrm{~mm}$ in diameter and was in the exact location of the cerebral hypothermic exposure. The cerebral cortex in several control animals was found to be necrotic in the area of hypothermia. In contrast, brain tissue in the experimental group of cats showed no sign of thermal injury to the brain. It was stated that in the experimental animals the omentum was "lightly adherent to the surface of the brain but could be peeled away easily." The presence of brain necrosis in the control animals and the absence of necrosis in the experimental animals were not discussed in the 1991 paper. A possible explanation could be that in the control animals, the hypothermic temperature applied prior to the opening of their dura and arachnoid destroyed BBB capillaries at the site of the hypothermic application. This could have caused brain tissue to become necrotic due to the loss of blood flow through damaged and non-functioning BBB capillaries caused by the hypothermia.

Brain tissue conditions were different in the experimental animals following their exposure to the hypothermic application. After their dura and arachnoid had been opened following the hypothermic application, the intact omental pedicle that had been lengthened surgically in these experimental animals was passed through the small frontoparietal craniotomy which allowed the omentum to be in direct contact with the underlying brain tissue. BBB capillaries that had been damaged by hypothermia in the control animals must also have occurred in the experimental group. This capillary damage would have led to a decrease in blood flow to the hypothermic damaged area of the brain. The decrease in blood flow through damaged BBB capillaries would have been compensated however, in the experimental animals as compared to the control animals because of the placement of the omental pedicle that had been directly positioned on the underlying brain tissue in the experimental group. Omental arteries originating from the omental pedicle would have had the ability to penetrate directly and deeply into the underlying brain [13]. This would have allowed increased blood flow to the hypothermic region in the experimental animals, but since there was no omental pedicle placed on the brain in the control animals, this group would not have experienced this increased cerebral blood flow to the brain. The additional source of blood to the hypothermic area in the experimental group flowing through arteries from the omental pedicle could explain why brain necrosis did not occur in this group as compared to the lack of increase blood flow in the control group.

In the 1991 paper [10] hypothermia was created at a temperature that was expected to produce vasogenic edema within the brain. As earlier reported [1], vasogenic edema produced by hypothermia 
normally reaches its maximal effect on the third day after hypothermic application. When the animals in the 1991 study were sacrificed 72 hours later, (3 days) following hypothermic application, no sign of vasogenic edema was reported. Tissue blocks were taken from all areas of the brain and dried in an oven at $65^{\circ} \mathrm{C}$ for $8-10$ days. Water concentration from individual cells throughout the brain was determined by weight-water content. Of significance was a complete lack of statistical difference between the water values in the control and experimental specimens (Wilcoxon's two sample rank sum test, $\mathrm{p}=$ 0.206). That the water content showed no change in any of the cells strongly suggests that the reason the omentum was reported to have failed to absorb vasogenic edema was because vasogenic had not been produced. The low temperature apparently was not low enough in the experiment to create vasogenic edema. The thermal conductivity created by the hypothermic application most likely failed to create a temperature that was low enough through the dural membrane to produce vasogenic edema.

\section{Conclusion}

It is difficult to accept the statement in the 1991 paper that "a pedicle omental graft failed to reduce vasogenic edema in an acute model and probably has no role in the acute management of brain edema." This statement seriously lessens the possibility that the omentum could have the capacity to absorb the vasogenic edema associated with a TBI. If the omentum could have the ability to absorb vasogenic edema following a TBI, this may well prove to be of significant clinical importance. This statement is based on the idea that the need to treat traumatic brain injuries will always occur and will probably continue in greater number over the years because of an expanding population. The United States Department of Health and Human Services has reported that 52,000 patients die annually (143 per day) from TBI's with 275,000 patients (733 per day) who require hospitalization. It therefore seems reasonable to suggest that the use of the omentum in the treatment of a TBI deserves present-day reevaluation. This will require studies be carried out in the laboratory and in the operating room. Such investigation appears advisable.

\section{Data acquisitions}

The material for the paper was published in 1991 [3].

\section{Declarations}

Ethics approval - No ethics approval is needed since experiment did not involve human participants and there was no ethics committee involved in animal research back in the 1980's.

Consent for publication - The manuscript does not contain any individual's data so this section is "non-applicable".
Availability of data and supporting materials section - The paper being presented is based on a paper listed as figure 1 in the reference section. The information in this present paper is simply an evaluation of their data.

Competing interests - The author H.S. Goldsmith has no competing interest in this paper.

Funding - No funding was necessary in the paper being presented.

Authors' contribution - H.S. Goldsmith is the single author

Acknowledgements - No acknowledgements are necessary

\section{References}

1. Michinaga S, Koyama Y (2015) Pathogenesis of brain edema and investigation into anti-edema drugs. Int J Mol Sci 16: 9949-9975. [Crossref]

2. Goldsmith HS (1974) Long term evaluation of omental transposition for chronic lymphedema. Ann Surg 180: 847-849. [Crossref]

3. Rajshekhar V, White LM, Harbaugh RE (1991) Acute effects of a pedicled omental graft on cold-induced brain oedema in cats. Acta Neurochir (Wien) 112: 136-140. [Crossref]

4. Schachtrup C, Lu P, Jones LL, Lee JK, Lu J, et al. (2007) Fibrinogen inhibits neurite outgrowth via B3 intigrin-mediated phosphorylation of EGF receptor. Proc Nat Acad Sci 104: 11814-11819. [Crossref]

5. Schachtrup C, Ryu JK, Helmrick MJ, Vagena E, Galanakis DK, et al. (2010) Fibrinogen triggers astrocyte scar formation by promoting the availability of active TGF-B after vascular damage. J Neurosci 30: 5843-5854. [Crossref]

6. Goldsmith HS, Steward E, Duckett S (1985) Early application of pedicled omentum to the acutely traumatised spinal cord. Paraplegia 23: 100-112. [Crossref]

7. Goldsmith HS, Saunders RL, Reeves AG, Allen CD, Milne J (1979) Omental transposition to brain of stroke patients. Stroke 10: 471-472. [Crossref]

8. Goldsmith HS, Bacciu P, Cossu M, Pau A, Rodriguez G, et al. (1990) Regional cerebral blood flow after omental transposition to the ischemic brain in man: A five year followup study. Acta Neurochir 106: 145-152. [Crossref]

9. Goldsmith HS (2011) A new approach to the treatment of Alzheimer's disease: the need for a controlled study. J Alzheimers Dis 25: 209-212. [Crossref]

10. Goldsmith HS (2014) Benefit of omental blood flow in Alzheimer's disease: effect on deteriorating neurons. J Alzheimers Dis 42 Suppl 3: S277-280. [Crossref]

11. Wei-Lie W, Shung-Qing X, Ming L, Xu-Ming H, Feng J (2010) Omental transposition following arachnoid excision to treat post-cerebral anoxia (cerebral palsy_ (chapter 13) IN: Goldsmith HS., Ed. The omentum: basic research and clinical applications, Woodbury, CT Cine-Med Publishing 167-172.

12. Goldsmith HS, Duckett S, Chen WF (1978) Prevention of cerebral infarction in the monkey by omental transposition to the brain. Stroke 9: 224-229. [Crossref]

13. Goldsmith HS (2010) Application of the omentum to the brain and spinal cord (Chapter 3) in: Goldsmith HS, ED, The omentum: basic research and clinical application, Woodbury, CT, Cine-Med Publishing 37-52.

Copyright: (C2017 Goldsmith HS. This is an open-access article distributed under the terms of the Creative Commons Attribution License, which permits unrestricted use, distribution, and reproduction in any medium, provided the original author and source are credited. 